\title{
Modelo para establecer la influencia de la responsabilidad social empresarial en el desempeño de los empleados de hoteles
}

DOI: 10.22403/UQROOMX/TYP10/01

\author{
Alejandro Alvarado-Herrera \\ Gegthsy Mariel Hernández González \\ Universidad de Quintana Roo \\ Unidad Académica Cozumel
}

Wh

\section{RESUMEN}

Uno de los mayores retos de las empresas turísticas en general, y las hoteleras en particular, es contar con estrategias y herramientas que promuevan y mejoren la participación y el desempeño de sus trabajadores, quienes son los responsables de ejecutar las funciones y procesos que permiten a la empresa generar, distribuir y comunicar valor. Por ello, y puesto que la responsabilidad social empresarial (RSE) o corporativa (RSC): I) tiene como uno de sus enfoques centrales las relaciones con los distintos grupos de interés de la organización (stakeholders); 2) ha demostrado generar importantes ventajas competitivas mediante el mejoramiento de su imagen y reputación, así como de una mayor identificación y satisfacción de sus clientes; 3 ) ha tenido un incremento en su aceptación e importancia para las organizaciones y la sociedad; y que, además, uno de los grupos de interés primario de la empresa son sus empleados, es necesario entender la posible influencia que sus percepciones en torno a la RSE ejercen en su comportamiento laboral. Dado que el tema ha sido poco estudiado, resulta oportuno determinar esos posibles efectos sobre la conducta, concretamente en la identificación, la motivación y la intención de permanencia laboral. Este trabajo propone un modelo teórico capaz de explicar las relaciones entre estos elementos, susceptible de ser contrastado a través de un sistema de ecuaciones estructurales (SEM, por sus siglas en inglés).

\section{Palabras Responsabilidad social empresarial, responsabilidad social \\ CLAVE corporativa, motivación, permanencia laboral, identificación empleado-empresa}




\section{Introducción}

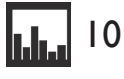

Algunas de las principales características de los entornos competitivos contemporáneos, como la fragmentación de los mercados, el acortamiento de los ciclos de vida de los productos, el cambio en los patrones de consumo de los individuos y la inestabilidad del mercado laboral (Jüttner y Wehrli, 1994), han conducido a que los gestores e investigadores de las ciencias económicoadministrativas consideren la creación y el desarrollo de relaciones estables entre las empresas y sus distintos grupos de interés (stakeholders) uno de sus principales afanes, ya que saben que esos stakeholders pueden afectar el logro de las metas de la organización o ser afectados por tal logro (Freeman y Reed, 1983; Jones y Wicks, 1999).

La idea de que los individuos que componen esos grupos premian a las empresas que se involucran en actividades de responsabilidad social empresarial (RSE) ha encontrado eco en la investigación científica y en la evidencia que la soporta, aun cuando los stakeholders provengan de esferas tan disímiles como la de los consumidores, los empleados o los inversionistas (Bhattacharya, Korschun y Sen, 2009). Esto, aunado a que en la actualidad la eficiencia económica de las empresas no garantiza su posicionamiento en un mercado (Lindgreen, Swaen y Maon, 2008), ha llevado a las empresas a efectuar cambios estructurales y de producción, y a realizar o apoyar actividades o causas sociales (Bigné, Currás y Sánchez, 2009; Vaca, Moreno y Riquel, 2007) en su búsqueda por alcanzar ventajas competitivas sostenibles (McWilliams, Siegel y Wright, $2006 a$ y b).

Ahora bien, pese a que diversos estudios han comprobado que la RSE percibida por los consumidores ejerce una influencia positiva y significativa sobre variables comportamentales como la intención de compra, la identificación del consumidor con la empresa, la reputación y la imagen de la firma,' los investigadores han llamado, cada vez más insistentemente, a examinar con mayor detalle a múltiples y distintos stakeholders (Bhattacharya, Korschun y Sen, 2009; Smith, 2003).

'Véase Alvarado-Herrera y Schlesinger, 2008; Bigné y Currás, 2008; Bigné et al., 2009; Kotler y Keller, 2006; Marín y Ruiz, 2007; Marín, Ruiz y Rubio, 2009; Sen y Bhattacharya, 200 I; Vaca, Moreno y Riquel, 2007. 
Como respuesta a esos llamados, y con la intención de contribuir al conocimiento de la RSE en general y del marketing de servicios turísticos en particular, se diseñó y se está llevando a cabo una investigación en la que se abordan las percepciones de la RSE por parte de distintos stakeholders, y su influencia en variables relativas al comportamiento de los sujetos, empleando como marco contextual el sector servicios, con énfasis en el subsector de servicios turísticos.

Este trabajo forma parte de esa investigación, y su objetivo principal es proponer un modelo teórico para determinar los posibles efectos, directos e indirectos, ejercidos por la RSE percibida por los empleados de hoteles sobre tres variables clave de su comportamiento laboral, a saber, I) su intención de permanencia, 2) su motivación y 3) su identificación con la empresa o laboral; modelo susceptible de ser contrastado a través de un sistema de ecuaciones estructurales (SEM, por sus siglas en inglés).

\section{Revisión de la literatura}

Responsabilidad social empresarial y desarrollo sustentable

La RSE ha sido estudiada desde diferentes perspectiva teóricas y, tal como exponen Bigné et al. (2007), el modelo más aceptado en la literatura especializada es el de Carroll (1979, I99 I y 1999), el cual propone cuatro dimensiones dependientes sucesivamente una de otra (Alvarado-Herrera y Schlesinger, 2008), y relacionadas con las expectativas de la sociedad respecto a que las empresas se comprometan de manera voluntaria y satisfagan aspectos económicos, legales, éticos y filantrópicos (también llamada dimensión discrecional) (Carroll, 1999). No obstante, las dimensiones antedichas no han podido ser confirmadas empíricamente de forma consistente, ya que cuando se ha intentado hacerlo discrepan los resultados obtenidos por los investigadores. ${ }^{2}$ Por consecuencia, los académicos han continuado haciendo llamamientos para desarrollar nuevos esquemas con los cuales medir el concepto.

En este sentido, una aproximación teórica alternativa que ha sido empleada para el estudio de la RSE es la ofrecida por el paradigma del desarrollo sustentable o sostenible (DS), según la cual la RSE es un reflejo de las mayores expectativas

\footnotetext{
${ }^{2}$ Véase Alvarado-Herrera, 2008;Alvarado-Herrera y Schlesinger, 2008; Currás, 2007; García de los Salmones, Herrero y Rodríguez del Bosque, 2005; Maignan, 200 I; Maignan y Ferrell, 2003.
} 
de ciudadanos, consumidores e inversionistas en cuanto al crecimiento económico, la cohesión social y la protección del medio ambiente (Comisión Europea, 200 I; Eberhard-Harribey, 2006). Esta perspectiva, además de tener un mejor ajuste conceptual al contexto del marketing de servicios turísticos -por tratarse del paradigma adoptado desde la década de los ochenta por la Organización Mundial del Turismo (ОМт, 2004)-, fue la empleada por Bigné et al. (2007) para establecer una definición específica de RSE para el contexto estudiado, la cual se introdujo en el XII Congreso de la Asociación Española de Expertos Científicos en Turismo,y es, precisamente ésta, la posición teórica asumida en el presente trabajo, por lo que se entiende, en consecuencia, que la RSE percibida (RSEP) es el:

Proceso y conjunto de actividades de marketing, de carácter estratégico y proactivo, que lleva a cabo una organización empresarial de servicios turísticos y que incorpora las preocupaciones sociales y medioambientales de sus stakeholders de forma que se minimice el daño y se maximice el impacto benéfico de la firma sobre la sociedad en el largo plazo [Bigné et al., 2007: I4].

\section{Identificación laboral}

En las ciencias económico-administrativas, el estudio de la identificación laboral (IL) o identificación organizacional (IO) se fundamenta principalmente en las teorías de la identidad social (TIS) (Tajfel y Turner, 1986) y de la autocategorización (TAC) (Turner et al., 1990), y reconocidos investigadores del área de comportamiento organizacional, como Ashforth y Mael (1989), han empleado las propuestas conceptuales de ambas teorías como punto de apoyo para avanzar en la comprensión de los comportamientos grupales dentro de la organización y la relación de éstos con respuestas consecuentes y reacciones individuales respecto a la misma, entre las que se encuentran la intención de permanencia (IP) y la motivación hacia el trabajo (MT).

La 10 es definida por Ashforth y Mael (1989:2I) como una "percepción de pertenencia o unidad con la organización", en tanto que Dutton, Dukerich y Harquail (1994:242) postulan que se trata del "grado en que un miembro de la organización se define a sí mismo a través de los mismos atributos que cree que definen a esa organización". Se puede decir que la IL es un tipo concreto de identificación social, en que el grupo o categoría social donde se ubica el 
sujeto es una organización (Mael y Ashforth, 1992), para la cual labora. Cuando el empleado compara subjetivamente los rasgos de identidad que percibe de la organización con los suyos, se puede generar un estado cognitivo de conexión o cercanía entre los mismos (IL), es entonces cuando la organización es psicológicamente aceptada como parte de la identidad personal del individuo (Scott y Lane, 2000).

\section{Modelo teórico}

La figura I muestra el modelo teórico propuesto en este estudio, el cual postula, basado parcialmente en Alvarado-Herrera (2008), Bigné et al. (2007) y EberhardHarribey (2006), que la RSE de las organizaciones hoteleras es percibida por sus empleados como un constructo multidimensional constituido por las dimensiones social, medioambiental y económica.

Asimismo,y con fundamento parcial en los planteamientos de Bhattacharya, Korschun y Sen (2009), Ashforth y Mael (1989), Dutton, Dukerich y Harquail (1994), Mael y Ashforth (1992) y Scott y Lane (2000), el modelo propone que las percepciones de los empleados en torno a la RSE de las organizaciones hoteleras para las que trabajan influyen en algunos de sus comportamientos, ya que la RSEp es capaz de producir en los empleados ciertos beneficios -psicológicos y simbólicos- que provocan su identificación laboral (IL), y a través de ésta se

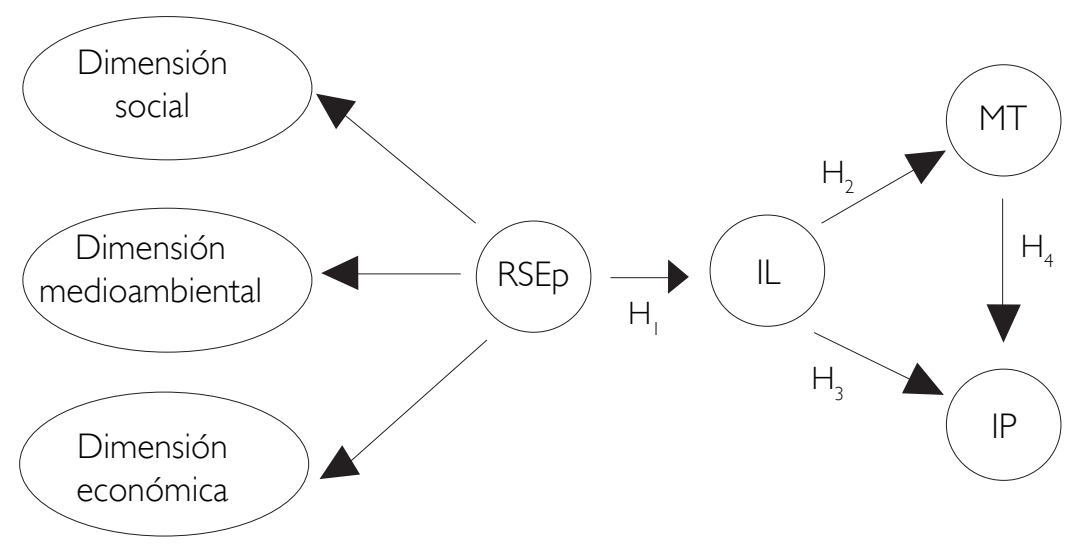

Figura I. Modelo propuesto 
tienen consecuentemente respuestas comportamentales más provechosas para la firma; en otras palabras, la IL es una variable mediadora entre la RSEp por los empleados de la empresa, su motivación hacia el trabajo y su intención de permanencia.

De esta forma, en nuestro modelo se establece que la percepción que tiene el empleado del grado en que la compañía cumple con las tres dimensiones del desarrollo sostenible (económica, medioambiental y social), y que cristaliza como constructo reflectivo de segundo orden en una percepción global de la RSE, es capaz de influir en respuestas positivas del individuo, como una mayor motivación hacia el trabajo y la intención de continuar en la compañía, por medio de una mejora en la identificación organizacional del sujeto.

Siguiendo los planteamientos teóricos apuntados, las hipótesis propuestas para su contrastación mediante SEM Son:

$\mathrm{H}_{1}$ : Las percepciones de los empleados de los hoteles acerca de la RSE de su organización influyen directa y positivamente en su identificación laboral.

$\mathrm{H}_{2}$ : La identificación laboral basada en la RSEp influye directa y positivamente en la motivación del empleado hacia su trabajo.

$\mathrm{H}_{3}$ : La identificación laboral basada en la RSEp influye directa y positivamente en la intención del empleado de permanecer en la compañía.

$\mathrm{H}_{4}$ : La motivación del empleado hacia su trabajo influye directa y positivamente en su intención de continuar trabajando en la compañía.

\section{Conclusiones y limitaciones}

Con la finalidad de contribuir al desarrollo de nuevos conocimientos en las áreas de la RSE y del marketing de servicios turísticos, y como parte de un proyecto de mayor envergadura, se llevó a cabo una investigación basada en la revisión de literatura relacionada con la temática y se propuso un modelo teórico, enfocado en los empleados como grupo de interés, capaz de determinar las influencias directas e indirectas ejercidas por las percepciones de la RSE sobre la intención de permanencia en la empresa, la motivación y la identificación organizacional. 
La revisión permitió establecer relaciones teóricas directas entre la RSEP y la identificación laboral,y entre ésta, la motivación hacia el trabajo y la intención de permanencia, por lo que se asume que la identificación laboral desempeñaría un papel como variable mediadora entre la primera y las dos últimas. También fue posible plantear que la motivación influiría directa y positivamente sobre la intención de permanencia del empleado en el hotel para el cual trabaja. El modelo propuesto cumple, en nuestra opinión, con las condiciones necesarias para ser contrastado mediante análisis factoriales confirmatorios de primero y segundo órdenes y sistemas de ecuaciones estructurales.

Ahora bien, dado que éste es un trabajo en curso, se reconoce que, pese a que hemos propuesto el modelo teórico, aún falta su contrastación empírica sometiendo a falsación las hipótesis que lo componen. De igual modo, cabe precisar que, hasta el punto que ha avanzado la presente investigación, y siguiendo una lógica confirmatoria, no se han encontrado fundamentos teóricos suficientes que hagan razonable plantear influencias directas entre las percepciones de los empleados respecto a la RSE de su organización y su motivación e intención de permanencia; sin embargo, no se descarta que tales fundamentos pudieran existir, por lo que se requiere profundizar en los estudios $y$, eventualmente, hacer alguna aproximación exploratoria a través de PLS.

Finalmente, aunque las limitaciones antedichas reducen la posibilidad de generalizar el resultado obtenido, consideramos que se ha conseguido un primer acercamiento al modelo, el cual contribuye, modesta pero ordenadamente, a la generación de los nuevos conocimientos buscados.

\section{Agradecimientos}

Los autores agradecemos los comentarios y la retroalimentación de los pares académicos a la versión preliminar de este trabajo, presentada en el Simposium Responsabilidad Social de las Organizaciones en el II Congreso Internacional Ciencias, Tecnologías y Culturas. 


\section{FUENTES CONSULTADAS}

\section{$\mid 6$}

Alvarado-Herrera, A. (2008). Responsabilidad social empresarial percibida desde una perspectiva sostenicéntrica, y su influencia en la reputación de la empresa y en el comportamiento del turista. Tesis doctoral. Valencia: Universitat de València.

Alvarado-Herrera, A. y M. Schlesinger (2008). "Dimensionalidad de la responsabilidad social empresarial percibida y sus efectos sobre la imagen y la reputación: una aproximación desde el modelo de Carroll”. Estudios Gerenciales, 24 (108), 37-59.

American Marketing Association (2004). "Marketing Redefined: Nine Top Marketers Offer their Personal Definitions". Marketing News, 38, 16. Ashforth, B. y F. Mael (1989). "Social Identity Theory and the Organization". The Academy of Management Review, I 4 (I), 20-39.

Bhattacharya, C. B., D. Korschun y S. Sen (2009). "Strengthening StakeholderCompany Relationships Through Mutually Beneficial Corporate Social Responsibility Initiatives". Journal of Business Ethics, 85, marzo, 257-272.

Bigné, E. y R. Currás (2008). “influye la imagen de responsabilidad social en la intención de compra? El papel de la identificación del consumidor con la empresa". Universia Business Review, 19, 10-23.

R. Currás e I. Sánchez (2009). "Brand Credibility in Cause-Related Marketing: the Moderating Role of Consumer Values". Journal of Product \& Brand Management, I8 (6), 437-447.

et al. (2007). "Desarrollo sostenible y responsabilidad social empresarial en el contexto del marketing de servicios turísticos: propuesta de una definición específica", en S. Antón y J. I. Pulido (eds.). Conocimiento, Creatividad y Tecnología para un Turismo Sostenible y Competitivo. XII Congreso de la Asociación Española de Expertos Científicos en Turismo. Tarragona: Asociación Española de Expertos Científicos en Turismo (Aecit)/Universitat Rovira i Virgili.

(2009). "Influencia de la percepción de la dimensión económica de la RSE sobre la satisfacción y la lealtad del consumidor". Revista de Responsabilidad Social de la Empresa, I, II7-I37.

Carroll, A. (1979). "A Three-dimensional Conceptual Model of Corporate Social Performance". Academy of Management Review, 4, 497-505. 
, (199I). "The Pyramid of Corporate Social Responsibility: Toward the Moral Management of Organizational Stakeholders". Business Horizons, 34 (4), 39-48.

, (1999). "Corporate Social Responsibility: Evolution of a Definitional Construct”. Business \& Society, 38 (3), 268-295.

Comisión Europea (200I). Libro verde: fomentar un marco europeo para la responsabilidad social de las empresas. Bruselas: Comisión de las Comunidades Europeas.

Currás, R. (2007). Comunicación de la responsabilidad social corporativa: imagen e identificación con la empresa como antecedentes del comportamiento del consumidor. Tesis doctoral.Valencia: Universitat de València.

Dutton, J., J. Dukerich y C. Harquail (1994).“Organizational Images and Member Identification”. Administrative Science Quarterly, 39 (2), 239-263.

Eberhard-Harribey, L. (2006). "Corporate Social Responsibility as a New Paradigm in the European Policy: How CSR Comes to Legitimate the European Regulation Process". Corporate Governance, 6 (4), 358-368. Freeman, R. E. y D. L. Reed (1983). "Stockholders and Stakeholders: A New Perspective on Corporate Governance". California Management Review, 25 (3), 88-I06.

García de los Salmones, M. del M., A. Herrero e I. Rodríguez del Bosque (2005). "Influence of Corporate Social Responsibility on Loyalty and Valuation of Services". Journal of Business Ethics, 6I (4), 369-385.

Jones, G. R. (200 I). Organizational Theory:Text and Cases. $3^{\mathrm{a}}$ ed. Texas: PrenticeHall.

Jones, T. M. y A. C. Wicks (I999). “Convergent Stakeholder Theory”. The Academy of Management Review, 24 (2), 206-22I.

Jüttner, U. y H.Wehrli (1994). "Relationship Marketing from a Value System Perspective”. International Journal of Service Industry Management, 5 (5), 54-73.

Keefe, L. M. (2004). “What is the Meaning of 'Marketing'?” Marketing News, 38, I7- 18.

Kotler, P. y K. Keller (2006). Dirección de marketing. I2ª ed. México: Pearson Educación.

Lindgreen, A., V. Swaen y F. Maon (2008). "Introduction: Corporate Social Responsibility Implementation”. Journal of Business Ethics, 85, 25I256. 
Mael, A. y E. Ashforth (1992). "Alumni and Their Alma Mater: a Partial Test of the Reformulated Model of Organizational Identification". Journal of Organizational Behavior, 13, 103-123.

Maignan, I. (200I). "Consumers' Perceptions of Corporate Social Responsibilities: A Crosscultural Comparison". Journal of Business Ethics, 30 (I), 57-72.

Maignan, I. y O. C. Ferrell (2003). "Nature of Corporate Responsibilities: Perspectives from American, French, and German Consumers". Journal of Business Research, 56 (I), 55-67.

Marín, L.y S. Ruiz (2007).““I Need you Too!' Corporate Identity Attractiveness for Consumers and the Role of Social Responsibility". Journal of Business Ethics, 7I (3), 245-260.

Marín, L., S. Ruiz y A. Rubio (2009). “The Role of Identity Salience in the Effects of Corporate Social Responsibility on Consumer Behavior”. Journal of Business Ethics, 84, 65-78.

McWilliams, A., D. S. Siegel y P. M. Wright (2006a). "Corporate Social Responsibility: Strategic Implications".Journal of Management Studies, 43 (I), I- 18.

— tives". Journal of Business Strategies, 23 (I), I-I 2.

OMT (2004). Declaraciones de la Organización Mundial de Turismo en relación con el desarrollo sostenible del turismo: conceptos y definiciones [en línea]. Disponible en: http://www.unwto.org/espanol/frameset/frame_ sustainable.html [2007, 24 de noviembre].

Scott, S. y V. Lane (2000). “A Stakeholder Approach to Organizational Identity”. The Academy of Management Review, I, 25 de enero de 2000, 49-62.

Sen, S. y C. Bhattacharya (200I). "Does Doing Good Always Lead to Doing Better? Consumer Reactions to Corporate Social Responsibility”. Journal of Marketing Research, 38 (2), 225-243.

Smith, N. C. (2003). "Corporate Social Responsibility: Whether or How?" California Management Review, 45 (4), 52-76.

Tajfel, H. y J. Turner (I986). "The Social Identity Theory of Inter-group Behavior”, en S.Worchel y L.Austin. Psychology of Intergroup Relations. Chicago: Nelson-Hall. 
Turner, J. et al. (1990). Redescubrir el grupo social. Una teoría de la categorización del yo. Madrid: Morata.

Vaca, R. M., M.J. Moreno y F. Riquel (2007).“Análisis de la responsabilidad social corporativa desde tres enfoques: stakeholders, capital intelectual y teoría institucional”, en J. C. Ayala Calvo y Grupo de Investigación FEDRA. Conocimiento, Innovación y Emprendedores. Camino al Futuro. XVII Jornadas Hispano-Lusas de Gestión Científica. Logroño:Universidad de La Rioja, 3|30-3|43. 
